\title{
User Experience Study of Multiple Photo Streams Visualization
}

\author{
Sam Zargham \\ Department of Electronics \\ University of Surrey \\ s.zargham@surrey.ac.uk
}

\author{
Janko Calic \\ Department of Electronics \\ University of Surrey \\ j.calic@surrey.ac.uk
}

\author{
David Frohlich \\ Digital World Research Centre \\ University of Surrey \\ d.frohlich@surrey.ac.uk
}

\begin{abstract}
With the expansion of digital photographic content stored online and concurrent proliferation of capturing devices, the management and visualization of personal photo collections have become very challenging tasks. In order to gain insight into novel ways of handling and representing large personal photo collections, this paper presents results of a user experience study into novel visualizations of multiple photo streams, sourced from different individuals or capture devices. A web-based application prototype was designed and implemented offering synchronized visualization of photo streams in a single- or multi-window display layout. An experimental study was conducted with 20 users, and the results demonstrate high user demand for concurrent presentation of multiple media streams as well as recommends methods for leveraging its potential.
\end{abstract}

Keywords: Photo sharing, multiple photo streams, presentation, user studies, social media

\section{INTRODUCTION}

Due to the proliferation of photo capturing devices, the volume of personal photos stored locally or hosted online has been increasing exponentially. The consumers cannot afford time to review their photo collections effectively. In addition to challenges of photo presentation, the emerging trend of Social Media over cloud based services plays an active role in shaping the current practices around personal photography.

In this paper, a web-based photo sharing application has been designed to study the practice of displaying and sharing personal photos in the social context. This study compares a single image presentation to a multiple concurrent presentation of different photo streams from different sources. The system utilizes a time-lapse presentation of multiple photo streams and enables fine-tuning of parameters such as desired presentation time, event change alerts and proportional transition interval to optimize the end-user experience. The user study has been conducted with two independent groups of friends and one family, targeting a wide range of usage contexts to improve understanding of the requirements around user practice in personal photography in a social context.

\section{RELATED WORK}

The processes of capturing, organizing, sharing and displaying personal digital photographs are becoming more challenging with the rapid evolution in imaging technology. The way people interact with photos has significantly changed. Personal computers, tablets, mobile phones and online repositories are the new media hubs of the photographic experience.

There have been numerous studies focused on the capture devices such as digital camera, camera phone (Ito, Okabe et al. 2005; Koskinen and Kurvinen 2005), passive devices (Ljungblad 2009; $\mathrm{Si}, 2009$ ) and audio cameras (Frohlich and Fennell 2007). Digital photos have been expanded from digital capture devices. Therefore, photo management tools have become popular. In order to organize digital photos, previous researchers exploited two main features of digital photos: Image contents and metadata which extracted from EXIF (Electronics and Association 2002).

The research presented in (Graham, Garcia-Molina et al. 2002; Loui and Savakis 2003) takes advantage of temporal metadata as the basis for organizing digital photographs. In addition, the location features inside of the EXIF data were also utilized in organizing photos (Naaman, Yeh et al. 2005; Cao, Luo et al. 2008). Another approach to facilitate organizing digital photographs was using optical metadata (Sinha and Jain 2008). Moreover, combination of metadata and image content 
features (Platt 2000; Cooper, Foote et al. 2005) has been applied for photo collections management. In addition to management, there has been a lot of effort put into visualization and presentation of personal photo collections. There have been various ways of presenting photographs: such as slideshow (Huiying, Qingming et al. 2010; Wang, Collomosse et al. 2010), collage (Diakopoulos and Essa 2005; Wang, Quan et al. 2006) and tiling (Ren, Calic et al. ; Calic, Gibson et al. 2007; Chu, Chen et al. 2007). Another technique that has been proposed to facilitate visualization and organization of personal photos was summarization (Kormann, Dunker et al. 2009; Sinha 2011).

Taking photographs seems no longer just an act of memory intended task but it has increasingly become a tool for communication (Van Dijck 2008). Therefore, some researchers have proposed tools to support sharing; see (Sarvas, Viikari et al. 2004; Ah Kun and Marsden 2007). In (Patel, Clawson et al. 2009), a collocated-synchronous mobile application for photo displaying and sharing has been implemented. In addition, (Jang, Yoon et al. 2009; Yang, Luo et al. 2011) have proposed novel techniques in order to organizing multiple photo streams for collocated events. However, these approaches just covered collocated events and did not propose intuitive visualizations of photos that were obtained from different sources. This shows there has been a dearth of research in managing and displaying multiple photo streams. Hence, we propose a novel photo visualization method aimed at the social use of multiple photo streams.

\section{DESIGN AND IMPLEMENTATION}

The central goal of the presented study was to gather requirements of a novel presentation of multiple photo streams. Therefore, two interfaces of a social media sharing website were built. This platform (See in Figure 1) took advantage of social and temporal metadata to facilitate the intuitive presentation of multiple photo streams. Photo streams are shown in chronological order based on the capture date and time taken from the EXIF (Electronics and Association 2002). During the slideshow in both interfaces, the information of each photograph such as date, time and photo owner is shown to support the narrative.

The main contribution of single window interface is to merge and show multiple photo streams of different users in one slideshow window. In multiwindow interface, instead of integrating photo streams of multiple people in one window, users can watch their photo streams concurrently in up to four slideshow windows. Each window is dedicated to an explicit person or source.

Additional functionalities such as proportional transition intervals, desired time and event informer have been integrated into this application as follows:

Proportional transition is designed to give a notion of time difference between slideshow intervals. This implies that the more time offset between two photos, the more delay should be reflected in the slideshow transition interval.

Desired time is introduced to let the user choose the overall time span of slideshow presentation. The transition between each slide is adjusted by the desired time duration that the user chooses to see all photo streams.

Event change informer based on time clustering from (Loui and Savakis 2003) is intended to inform user when an event is changed by displaying the message: "Event Changed".

The system is implemented as a client-server model. The implementation layers comprise the data, application, presentation and client layer. Client access the system by web browser with client layer. The presentation layer contains the user interface of the system. This layer has been coded using HTML. Images and information about them has been stored in the data layer with Microsoft SQL Server. In the application layer, there is a set of components for mediating the presentation layer. This means that the core and logic of application is in this layer. Upload management, search manager, metadata EXIF extractor, proportional transition logic, and event informer and desired time manager all have been coded by $\mathrm{C \#}$ in this layer.

\section{METHODOLOGY}

The main goal of this study was to understand how a photo streaming service fits into the social lives of close friends or family. Once the system was constructed it was tested by deploying it to 20 users in three distinct groups mainly from the UK. (See in Table I)

Three groups were recruited for this study. The first group was comprised of close friends who were expected to upload the photographs of their life into the system. The user's age was in a range between 23 and 30 years and they were all university graduates.

Second group of friends shared their photographs over a 48 hours period in pre-planned events. The participant's age was between 25 to 32 years and they all were graduate students and postgraduate researchers. During the experiment, some participants were in different cities, while the majority of the group was in Guildford, UK.

The third group was a family whose members were located in England, and two overseas countries, one in America and one in continental Europe. The UK based members of the family: father, mother and a 5 years old child were living in a same place and they took a photo journal for 72 hours. The 


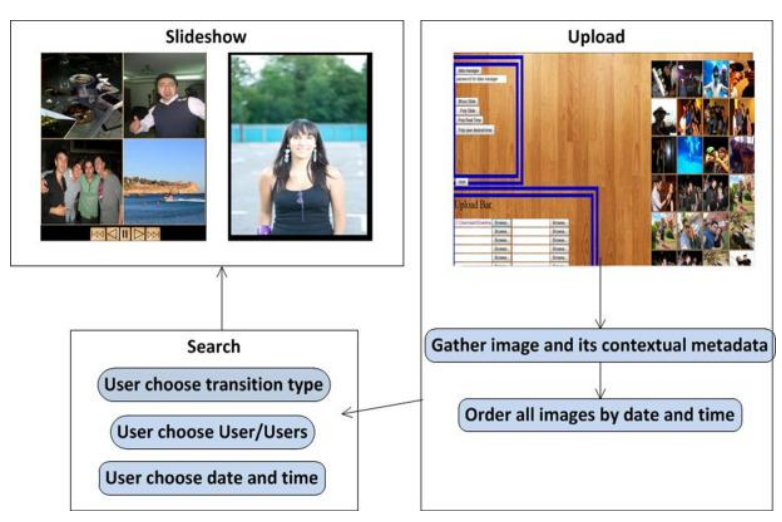

Figure1: Visualization of single- and multi-window interfaces.

niece and grandparents were living in different countries and took pictures during the same 72 hour period. At the end of the period, a grandparent and the core family were reunited in the UK. The ages were varied between 5 to 65 years old.

Semi structured interviews technique was chosen to gather information. The goal was general and qualitative information with enough depth was needed.

All interviews were recorded with video camera and took around 45 minutes each. Table I has shown the summary of the user group's information.

\section{RESULTS}

In the following section we describe the finding from our study. As mentioned earlier, we interviewed participants about how these two photo streaming interfaces fits into their life. Having performed data gathering sessions, inductive qualitative analysis was performed. Here we first present a description of the need for visualization and organization of multiple photo sources. Then, we continue break results following user's practices and motivations with the system with following themes of use.

Table I: Participant's Information

\begin{tabular}{|c|l|l|l|}
\hline & Group1 & Group2 & Group3 \\
\hline $\begin{array}{c}\text { Duration of } \\
\text { photo } \\
\text { collection }\end{array}$ & $\begin{array}{l}\text { Whole Life } \\
\text { Pictures }\end{array}$ & $48 \mathrm{~h}$ & $72 \mathrm{~h}$ \\
\hline Relation & $\begin{array}{l}\text { Close } \\
\text { friends }\end{array}$ & $\begin{array}{l}\text { Close } \\
\text { friends }\end{array}$ & Family \\
\hline Age Range & $23-30$ & $25-32$ & $5-65$ \\
\hline $\begin{array}{c}\text { Number of } \\
\text { Participants }\end{array}$ & 5 & 10 & 5 \\
\hline
\end{tabular}

\subsection{Multiple Photo Sources}

Most of the participants had different digital capture devices such as camera phone and standalone camera. This theme present in the data was the existence of multiple photo sources from different cameras to pictures that has been collected from social networks.

In family group, they had one shared digital camera at home for both mother and father. Daughter had her own camera and grandparent recently moved into using digital camera. The niece used SLR camera and camera phone. Mother used the shared digital camera and father took pictures with camera phone.

\begin{abstract}
Father stated: "The pictures of all cameras belong to the family. It is not important who with what device took a picture. However, sometimes we are too lazy to manage the pictures and we leave it as it is." He also added: "Because all pictures belong to the family, sometimes I want to filter our pictures based on capture devices."
\end{abstract}

AR a member of group two like most of the other participants in this group used her camera phone alongside her digital camera.
She said: "I wanted to take pictures of the cake. But, suddenly, the digital camera battery died. I compensated that by taking pictures with my camera phone. Camera phone is more accessible. Moreover, I took pictures with standalone digital camera when I needed to collect better quality images ".

The same, in group one, participant's photographs were combination of digital camera and camera phone. Therefore, it can be concluded from these discussions that participants are already using multiple cameras to generate streams representing their life experiences. Because current technology is individual user-centred, it tends not to integrate or combine these streams in any meaningful way. Furthermore, different devices have had different usage. Quality in camera phones never has been the same as traditional cameras but they were more accessible. Hence, participants did not want to combine their camera phone pictures with their digital camera pictures. However, in some cases when the camera phone pictures completed the story of their digital camera, there was a huge interest.

Another source of personal photo acquisition was social network websites.

GB a member of group one, said: "I am not the person who takes pictures, my friends usually 
take photograph and share it on Facebook with me".

All members of group one considered their tagged pictures in Facebook as their own photographs. Therefore, In addition to acquisition of images with multiple cameras, social network pictures considered as an alternative way of collecting photographs.

\subsection{Collocated Experience}

Across all three groups, watching collocated experience with both interfaces was motivating. The main contribution of single window interface was showing of one or different users pictures in one slideshow window. Single window interface surprised people when the taken photo streams were for the same time and place (collocated). The reason was completeness of an event. For example: in the birthday party, in the group two, participants believed they have not missed any single moment and scene of birthday.

$A M$ and GB two members of a group one were in a ski trip. Both were surprised when they saw that event and believed they discovered new information from the same trip that they were in. Again, two participants in the group one, AM and LK, have been in Spain for Christmas holidays. The completeness of an event from different photographers led to not missing any scene and face.

Multiple windows interface had up to four separated slideshow windows for each social for presenting the several photo streams. The same as single window interface, it was worthy in watching of collocated experience. Study showed that it was easier to understand who took the picture and participants can review the story effortlessly. The only problem was that images were smaller. This issue never mentioned when participants watched their pictures in big screen.

\subsection{Remote Experience}

An additional theme present in the data was the importance of remote experience in sharing behaviour.

Results have shown that unlike multi windows interface, single window interface did not surprise people when the photo streams were for same time but different place (remote). The study has shown although participants liked to know what happened to others while they were doing something else in different place at the same time, the unrelated events appearance changed the mood of participant. Most of them believed that changing from collocated to remote state was intrusive. However, they never denied the interest in awareness of other members.

AM and LK, members of group one were enjoying the photo streams of Spain Christmas trip and meanwhile, GB another member of a group one photo in UK appeared. The awareness was pleasant. Conversely, the pictures from another event in the middle of the main event were disturbing. In another case, those participants who were in birthday party suddenly saw a picture of TV and food from a friend at his home and they found it discouraging.

SO a member of group two said: "I like the awareness of single window interface. However, still I don't like the feeling when another unrelated picture interrupts my mood".

In multiple windows interface, participants never mentioned the problem of intrusiveness. Those participants who were together during the capture could follow the story of themselves while the story of other participants who were not with the others was without any interruption.

\begin{abstract}
The niece who is living in America said: "when I was seeing the pictures, I noticed that I feel more comfortable with multiple windows interface because the other pictures were from somewhere else and I could distinguish others photography experiences easily".
\end{abstract}

\subsection{Redundancy}

Redundancy happened in both single and multiple windows interface when the amount of some participant's pictures was much greater than others. The problem was when one person had large amount of photos in overall or in one event and following that, the slideshow became boring. Consequently, participants could not see their own collocated and remote experiences. This happened in the group one. The reason was that they uploaded their life pictures and there was not any pre-planned event to obtain pictures from the same time.

$\mathrm{AH}, \mathrm{LK}$ and GB members of group one commented that for a long period of time they just saw pictures of $A M$ and that was discouraging.

LK said: "AM had around 100 pictures. I am interested in watching her pictures form past but not all of them with people who I do not have any clue who they are".

Results showed Participants wanted to know while they were in an event, what others were doing (remote) or what happened between them (collocated). One solution to tackle this problem is summarization of redundant streams based on reviewer, type of event (collocated or remote) and appealing features.

\subsection{Functionalities of slideshow}

By enabling the proportional transition function into the system, user had this opportunity to review the 
slideshow by automated transition interval. When the date and time taken of consecutive pictures were close, the interval was less and when the date and time taken of two following pictures were far away the slideshow interval was more. The maximum interval is 5 seconds for the transition. On the other hand, the minimum interval is 3 milliseconds. However, in ambient mode, the slideshow interval is equal to differences between real date/time of pictures taken.

Most participants seemed to feel that proportional transition provides the sense of time between slides. This offered users better communication with pictures. Moreover, participants believed that they can understand when the event has been changed and how far the next event approximately was.

\footnotetext{
Mother of the family said: "night finished and we did not take any picture until morning. There was a delay in transition between night and morning. After that, grandmother was the one who took the first picture in the early morning. This function left more room for reflection about the time".
}

When we asked in what situation proportional transition was the best, MS said that it is a kind of smart system, which gives him the notion of time and feeling the change of event. In continues events, pictures have been taken in burst. Therefore, the speed was fast and he felt it was a movie. Similarly, TR a member of group two commented that she prefers this function in continues events rather than discrete events. However, she did not deny her interest in sensing the time.

Another function, event informer, clusters images and informs user by text "event changed".

\footnotetext{
SK mentioned: "proportional transition function let you feel the end of the event. Moreover, in event informer, I never had the perception about the time between intervals during the slideshow. Proportional transition function was more natural".
}

The system also supports reviewing pictures relying on desired time function. In such a function, the streams are shown over the time that user is chosen. During watching photo sets, this function presented participants sense of sentimentality in short period of time. On the other hand, this was valuable when it is used as a digital photo ambient display for longer time.

AR, a participant of group two said that she usually does not have time to review her whole pictures. Therefore, she was the fan of this function. Moreover, HF another member of group two said:

"Fast change reminds me what happened between our lives. This is what I wanted from this feature. It is fast way of remembering our past".

Another participant, PM, proposed to combine two proposed functions desired time and proportional transition to have the sense of time between images at the side of desired time.

LK, proposed when the shared moments appear, set delay and compensate it somewhere else such as not presenting the all unshared moments.

\section{CONCLUSION}

In this study we examined user experience of concurrent presentation of multiple photo streams using two interfaces. The single-window interface was proven to be suitable in combining the pictures of one event that occurs at the same time and same place. On the other hand, the single window interface was disruptive if the photo streams showed events from different places that happened at the same time (remote experience). Nevertheless, the multi-window interface enabled good awareness of represented events and was well received in both remote and collocated experience. The main hindrance in both systems was that often too many pictures would come from a single user, which hindered success of collocated or remote experience. One solution to overcome this problem is summarization of redundant photo streams, which will be the focus of our future work. In addition to joint summarization of multiple photo streams, an algorithm for dynamic optimization of time intervals will be studied. Finally, a novel algorithm for joint optimization of photo retargeting and window layout will be designed. All of the three algorithms are mutually dependent, so a joint global optimization system will be developed, offering a novel visualization and interface that adapts to the demands of the user, its content and social neighbourhood.

\section{REFERENCES}

Ah Kun, L. M. and G. Marsden (2007). Co-present photo sharing on mobile devices, ACM.

Calic, J., D. P. Gibson, et al. (2007). "Efficient layout of comic-like video summaries." Circuits and Systems for Video Technology, IEEE Transactions on 17(7): 931-936.

Cao, L., J. Luo, et al. (2008). Annotating collections of photos using hierarchical event and scene models, IEEE.

Chu, W. T., J. C. Chen, et al. (2007). "Tiling slideshow: An audiovisual presentation method for consumer photos." Multimedia, IEEE 14(3): 36-45. 
Cooper, M., J. Foote, et al. (2005). "Temporal event clustering for digital photo collections." $\mathrm{ACM}$ Trans. Multimedia Comput. Commun. Appl. 1(3): 269-288.

Diakopoulos, N. and I. Essa (2005). Mediating photo collage authoring, ACM.

Electronics, J. and I. T. I. Association (2002). "JEITA CP-3451 Exchangeable image file format for digital still cameras: Exif Version 2.2." Japan Electronics and Information Technology Industries Association.

Frohlich, D. and J. Fennell (2007). "Sound, paper and memorabilia: resources for a simpler digital photography." Personal and Ubiquitous Computing 11(2): 107-116.

Graham, A., H. Garcia-Molina, et al. (2002). Time as essence for photo browsing through personal digital libraries, ACM.

Huiying, L., H. Qingming, et al. (2010). Attention Based Album Slideshow. Image and Video Technology (PSIVT), 2010 Fourth Pacific-Rim Symposium on.

Ito, M., D. Okabe, et al. (2005). Personal, portable, pedestrian: Mobile phones in Japanese life, The MIT Press.

Jang, C., T. Yoon, et al. (2009). A New Clustering Methodology for Group Photos Taken by Multiple Travelers, IEEE.

Kormann, D., P. Dunker, et al. (2009). "Automatic Rating and Selection of Digital Photographs." Semantic Multimedia: 192-195.

Koskinen, I. and E. Kurvinen (2005). "Mobile multimedia and users: On the domestication of mobile multimedia." Telektronikk 101(3/4): 60.

Ljungblad, S. (2009). Passive photography from a creative perspective: "If I would just shoot the same thing for seven days, it's like... What's the point?". Proceedings of the 27th international conference on Human factors in computing systems. Boston, MA, USA, ACM: 829-838.

Loui, A. C. and A. Savakis (2003). "Automated event clustering and quality screening of consumer pictures for digital albuming." Multimedia, IEEE Transactions on 5(3): 390-402.

Naaman, M., R. B. Yeh, et al. (2005). Leveraging context to resolve identity in photo albums, IEEE.
Patel, N., J. Clawson, et al. (2009). "Mobiphos: A study of user engagement with a mobile collocatedsynchronous photo sharing application." International Journal of Human-Computer Studies 67(12): 1048-1059.

Platt, J. C. (2000). "AutoAlbum: Clustering digital photographs using probabilistic model merging." cbaivl: 96.

Ren, K., J. Calic, et al. User study of the free-eye photo browsing interface, IEEE.

Sarvas, R., M. Viikari, et al. (2004). MobShare: controlled and immediate sharing of mobile images, ACM.

$\mathrm{Si}$, (2009). Fixed in time and "time in motion": mobility of vision through a SenseCam lens. Proceedings of the 11th International Conference on Human-Computer Interaction with Mobile Devices and Services. Bonn, Germany, ACM: 1-10.

Sinha, P. (2011). Summarization of archived and shared personal photo collections, ACM.

Sinha, P. and R. Jain (2008). Classification and annotation of digital photos using optical context data, ACM.

Van Dijck, J. (2008). "Digital photography: communication, identity, memory." Visual Communication 7(1): 57.

Wang, J., L. Quan, et al. (2006). "Picture collage."

Wang, T., J. Collomosse, et al. (2010). "Stylized ambient displays of digital media collections." Computers \& Graphics.

Yang, J., J. Luo, et al. (2011). Photo stream alignment for collaborative photo collection and sharing in social media, ACM. 